\title{
Zum Umgang mit Intersex: Gibt es Wege jenseits der Zuordnung des «richtigen Geschlechts»?
}

\section{Nikola Biller-Andorno}

Prof. Dr. Dr. med.

Nikola Biller-Andorno

Ethik-Zentrum der Universität Zürich

Zollikerstrasse 115

CH-8008 Zürich

Tel. 0446348380

Fax 0446348389

biller-andorno@ethik.unizh.ch
Dieser Fall eines fünfjährigen «Knaben» mit Genotyp 46XX und adrenogenitalem Syndrom (AGS) ist in der Tat von erheblichem medizinethischem Interesse: Die anstehenden Entscheidungen bezüglich schwerwiegender medizinischer Eingriffe, wenngleich ohne vitale Indikation, betreffen ein nichteinwilligungsfähiges Kind aus einem anderen Kulturkreis und werden erheblichen Einfluss auf die spätere Lebensweise und Lebensqualität, ja sogar das Selbstverständnis des Kindes haben.

Geht es bei den zu treffenden Entscheidungen jedoch allein um die Abwägung zwischen Fertilitätsverlust (im Falle einer Zuordnung zum männlichen Geschlecht bei Entfernung von Uterus und Ovarien sowie späterem Testosteronersatz und Hodenprothesen) und einem Wechsel der bisherigen Geschlechtsorientierung (im Falle einer Zuordnung zum weiblichen Geschlecht mit Klitorisreduktion und Neubildung der Vagina), wie es die wohlüberlegte und ethisch versierte Darstellung des Falls nahelegt? Ist der Frage nach der Zuordnung des «richtigen Geschlechts» nicht die Frage voranzustellen, unter welchen Voraussetzungen eine solche Zuordnung überhaupt vorgenommen werden soll?

Die Falldarstellung erwähnt zwar eine Verschiebung der operativen «Korrektur» als «theoretische» Option, die die Einbeziehung des dann Jugendlichen in den Entscheidungsprozess ermöglicht, diskutiert aber nicht die Möglichkeit des Aufwachsens als Intersex. Die möglichst rasche und unzweifelhafte Zuordnung zum weiblichen oder männlichen Geschlecht scheint so offensichtlich im Kindeswohl zu sein, dass der Respekt vor der Autonomie des Betroffenen dahinter an Bedeutung zurücktritt und andere Wege gar nicht diskutiert werden.

Dieser Engführung liegt eine binäre Geschlechtskonzeption zugrunde, die aber im strikten Sinne weder genotypisch noch phänotypisch, noch in sozialer Hinsicht gegeben ist. Während anderen Kulturen der Umgang mit einem «dritten» Geschlecht nicht unvertraut ist, scheint es eine Spezifität unserer gegenwärtigen Kultur zu sein, die Erscheinung von Intersex als korrekturbedürftige Abweichung von der Norm zu betrachten. Ein drittes Geschlecht ist bei uns derzeit nicht «gesellschaftsfähig», wie es die Leitlinie der Deutschen Gesellschaft für Urologie zu Störungen der sexuellen Differenzierung (1999) formuliert. Gleichwohl erwähnen Leitlinien wie auch medizinische Literatur inzwischen zumeist die Forderung kritischer Stimmen, Geschlechtszuordnungen bis zur Einwilligungsfähigkeit zurückzustellen und Intersex als drittes Geschlecht anzuerkennen, wenn auch der Verweis auf diese Position in der weiteren Argumentation in der Regel folgenlos bleibt.

Aber kann denn das Kindeswohl so ohne weiteres für eine ärztliche «Vereindeutigung» des Geschlechts in Anspruch genommen werden? In der Literatur wird ein Prozentsatz von 15\% intersexueller Erwachsener genannt, die eine Verlegung von geschlechtszuweisenden Operationen ins Erwachsenenalter bzw. ein drittes Geschlecht befürworten. Stellungnahmen aus Patientenperspektive berichten von Traumatisierungen durch wiederholte Eingriffe und die Pathologisierung ihres Körpers, insbesondere ihrer Genitalien, sowie das Aufzwingen einer Norm anstelle von Toleranz für ihre körperliche Erscheinung.

Es gibt auch Stimmen, die die Zuordnung des männlichen oder weiblichen Geschlechts prinzipiell für den falschen Weg halten, unabhängig von der Frage des Wohls des Kindes bzw. des späteren Erwachsenen. Das Intersex per se sei kein pathologischer, behandlungsbedürftiger $\mathrm{Zu}$ stand, und «Heilung» bestünde vielmehr in der Überwindung von Isolation und Ausgrenzung sowie in der Akzeptanz des eigenen, intersexuellen Körpers. Problematisch sei nicht das Phänomen des Intersex, sondern die gesellschaftliche Reaktion.

Aber selbst wenn man sich eine offenere gesellschaftliche Einstellung gegenüber Intersex wünschen mag, die den Betreffenden ein Leben ohne Infragestellung und Zurückweisung ermöglicht - was heisst das für die individuelle Behandlung angesichts der Realitäten unserer gegenwärtigen Gesellschaft? Sollte man im Falle 
des fünfjährigen Knaben zusehen, wie die Brustentwicklung beginnt und er in seiner körperlichen Erscheinung zunehmend von seiner Umwelt hinterfragt wird? Die Frage, ob und in welchem Masse es Aufgabe der Medizin ist, Korrekturen körperlicher Konditionen vorzunehmen, die nur oder vornehmlich aufgrund der sozialen Reaktion ein Leiden darstellen, ist aus der Debatte um Eingriffe bei Minderwuchs, Segelohren oder kleinen Brüsten sattsam bekannt. Die Antworten variieren ebenso wie die zugrundeliegenden Krankheitsbegriffe und Auffassungen von der Rolle der Medizin. Sicher ist aber, dass es im Einzelfall durchaus grausam sein kann, gesellschaftliche Erziehungsmassnahmen auf dem Rücken (nicht- oder nur begrenzt einwilligungsfähiger) Individuen austragen zu wollen.

Was im Wohle des Kindes liegt, muss also eine individuelle Entscheidung bleiben. Die Falldarstellung beschreibt hier wichtige Aspekte in der Gestaltung des Prozesses, darunter das Vertrautmachen der Familie mit der Situation, das Heranziehen externer Übersetzer, die Diskussion der Frage, inwieweit das Kind involviert werden kann, sowie die Exploration des kulturellen Kontexts.
Als Lösung schiene mir - angesichts des relativ geringen Schadens/Risikos im Falle des Aufschiebens einer Operation und angesichts der noch nicht zufriedenstellenden Datenlage bezüglich der Auswirkungen der jeweiligen Eingriffe auf die Lebensqualität der Betroffenen folgende Herangehensweise erwägenswert: Dem Kind könnte derzeit durch Hormongaben eine Weiterentwicklung in der Knabenrolle ermöglicht werden, ohne durch eine Operation bereits irreversible Fakten zu schaffen. Damit kann, zumindest vorläufig, sowohl ein endgültiger Fertilitätsverlust als auch ein Wechsel der aktuellen Geschlechtsorientierung vermieden werden. Insbesondere könnte der «Junge» später selbst entscheiden, wie er seine geschlechtliche Identität gestalten möchte. Hierbei wären eine jeweils altersgemässe Aufklärung und eine psychologische Begleitung hilfreich, die zur Vorbereitung der später anstehenden eigenen Entscheidung beitragen kann. Auf diese Weise könnte die endgültige «Zuordnung» eines Geschlechts durch Arzt und Eltern vermieden werden, ohne das Kind den Belastungen einer offensichtlichen Existenz als Zwitter auszusetzen. 